\title{
E-Salud en Medicina del Trabajo como Oportunidad de Mejora en la Atención a la Enfermedad Profesional
}

\section{E-Health in Occupational Medicine as an Opportunity to Improve Care of Occupational Diseases}

\author{
D. Jerónimo Maqueda Blasco \\ Director de la Escuela Nacional de Medicina del Trabajo \\ Instituto de Salud Carlos III. \\ Madrid. España
}

El actual desarrollo de las Tecnologías de la Información (TIC) y Comunicación aplicadas a la práctica de la medicina, conocida con el término de $e$-Salud permiten que servicios no especializados dispongan de asesoramiento en diferentes especialidades médicas.

La aplicación de la Telemedicina ha sido evaluada en diferentes ámbitos clínicos como la dermatología y la neurología, en este sentido destaca el informe elaborado por la Agencia de Evaluación Tecnologías Sanitarias del ISCIII (Tele-dermatología, Revisión sistemática y evaluación económica), que pone de manifiesto un alto nivel de evidencia científica y grado de recomendación, en los siguientes aspectos del manejo clínico de este tipo de patología:

- Reducción del número de consultas hospitalarias (nivel de evidencia Ib y Grado de Recomendación A).

- Desde la perspectiva institucional la tele-dermatología resulta más económica que la asistencia convencional (nivel de evidencia IIb y Grado de Recomendación B).

- La tele-dermatología ha obtenido niveles de satisfacción elevado entre los pacientes atendidos (nivel de evidencia Ib y Grado de Recomendación A).

- La tele-dermatología ha obtenido niveles excelentes de satisfacción entre lo dermatólogos que la practican (nivel de evidencia Ib y Grado de Recomendación A).

Según este mismo informe la tele- dermatología ha obtenido niveles de satisfacción significativamente superiores a la asistencia convencional entre los médicos de atención primaria usuarios de esta metodología (nivel de evidencia Ib y Grado de Recomendación A).

El Sistema de Enfermedades Profesionales, debe saber trasladar a su práctica experiencias de éxito en otros Sistemas, en particular del Sistema Nacional de Salud (SNS).

El desarrollo de estrategias de e-Salud en Enfermedades Profesionales se alinea e incrementa la factibilidad de las nuevas políticas tanto Nacionales como Europeas en materia de Enfermedades Profesionales y Prevención de Riesgos Laborales, principalmente como instrumento facilitador de una mayor presencia del Sistema Nacional de Salud en estos ámbitos.

La Estrategia Europea de Salud y Seguridad en el Trabajo insta a los Estados Miembros en su punto 5.1. sobre incremento de la eficacia preventiva de la vigilancia de la salud a que los sistemas sanitarios nacionales desempeñen un papel más activo integrando, entre otras cosas, acciones de sensibilización de los médicos en lo que respecta a los antecedentes y a las condiciones de trabajo de sus pacientes.

Esta "participación activa" del Sistema Nacional de Salud esta también reclamada por el RD 1299/2006 que contempla diferentes medidas tendentes a la mejora de la notificación, calificación e información de enfermedades profesionales en las que concurre el Sistema Nacional de Salud.

En este sentido el artículo 5 relativo a la comunicación de las enfermedades que podrían ser calificadas como profesionales establece la obligación de los facultativos del Sistema Nacional de Salud de comunicar, a través de los órganos competentes de las CCAA el conocimiento de las enfermedades incluidas en el anexo I o II de dicho RD y cuyo origen profesional se sospeche. 
Esta nueva obligación de los facultativos del Sistema Nacional de Salud y en especial en el Nivel de Atención Primaria, por principio de correspondencia, ebe de acompañarse del desarrollo de medidas facilitadoras que enraizadas en el propio SNS proporcionen un servicio directo que contribuya al mejor manejo clínico de la enfermedad y a la identificación de la enfermedad profesional.

El desarrollo de la e-Salud en Enfermedades Profesionales supone, además de la adecuación al progreso, la mejora de la atención integral al trabajador, al permitir proporcionar servicios de asesoramiento especializado al SNS, tanto en los niveles de atención primaria como hospitalaria, en el ámbito del diagnóstico y valoración médica de las enfermedades profesionales al facilitar el establecimiento de relaciones entre Unidades Especializadas en Enfermedades Profesionales con: Servicios de Prevención de Riesgos Laborales, MATEPS y Equipos de Valoración de Incapacidades, dentro del nuestro Sistema de Seguridad Social y del Sistema Nacional de Salud, tanto en los niveles especializados como en el nivel de Atención Primaria

El desarrollo de la e-Salud en Enfermedades Profesionales es un medio para impulsar el trabajo en Red mediante la vertebración de nodos clínicos en el apoyo al manejo clínico de la enfermedad profesional (dermatología, neurología, neumología, toxicología) y nodos epidemiológicos en el apoyo a la programación preventiva.

Las estrategias de e-Salud supone una oportunidad innovadora en la mejora del manejo de la enfermedad profesional, así como la valorización de investigación clínica, epidemiológica y tecnológica.

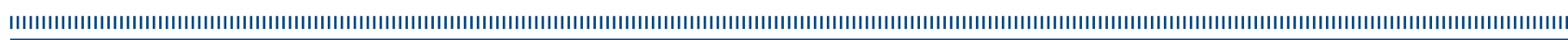

